\title{
Aplicação da Ressonância Magnética Nuclear no Estudo de Constituintes de Cannabis
}

\author{
Gabriel S. Ferreira, Júlia A. Leite, Bianca B. Merlo, Valdemar L. Junior, \\ Wanderson Romão \& Alvaro C. Neto
}

\begin{abstract}
A planta Cannabis apresenta uma classe característica de constituintes chamados canabinoides, os quais possuem propriedades psicotrópicas, e por esta razão, são de grande interesse forense. O isolamento desses compostos presentes nas drogas ilícitas (maconha, haxixe, etc.) preparadas a partir da Cannabis se constitui em uma difícil tarefa. Neste trabalho, as técnicas de Cromatografia em Coluna, Ressonância Magnética Nuclear (RMN) e Cromatografia Gasosa acoplada à Espectrometria de Massas (CG-EM) foram empregadas no isolamento e identificação de canabinoides presentes em amostras de maconha apreendidas pela Polícia Civil do Estado do Espírito Santo (PC-ES). Estas foram extraídas por maceração em metanol, analisadas por RMN de $1 \mathrm{H}$ em CDCl3 e, ao final, concentradas em um extrato bruto de maconha que foi submetido à Cromatografia em Coluna em sílicagel com fase móvel de éter de petróleo:éter etílico 8:2, coletando-se 52 frações, as quais foram então agrupadas em cinco frações finais após análise de placas de Cromatografia em Camada Delgada (CCD). Essas cinco frações foram submetidas à CG-EM e apenas as Frações 2 e 3 apresentaram o canabinoide identificado como Canabinol (CBN) em sua forma pura, o que foi comprovado pela análise do mesmo por RMN.
\end{abstract}

Palavras-chave: Maconha; Cannabis; Ressonância Magnética Nuclear, Canabinol.

Cannabis plant presents a characteristic class of constituents called cannabinoids, which have psychotropic properties, and for this reason they are of great forensic interest. The isolation of these compounds present in the illicit drugs (marijuana, hashish, etc.) prepared from Cannabis is a difficult task. In this work, Column Chromatography, Nuclear Magnetic Resonance (NMR) and Gas Chromatography coupled to Mass Spectrometry (GC-MS) techniques were used in the isolation and identification of cannabinoids present in marijuana samples seized by the Civil Police of Espírito Santo State (PC-ES). These were extracted trough maceration in methanol, analyzed by $1 \mathrm{HNMR}$ in $\mathrm{CDCl} 3$, and finally concentrated in a crude marijuana extract, which was subjected to silica gel column chromatography with mobile phase of petroleum ether:ethyl ether 8:2, collecting 52 fractions, which were then grouped into five final fractions after analysis of Thin Layer Chromatography (TLC) plates. These five fractions were submitted to GC-MS and only Fractions 2 and 3 showed the cannabinoid identified as Cannabinol $(\mathrm{CBN})$ in its pure form, which was evidenced by NMR analysis.

Keywords: Marijuana; Cannabis; Nuclear Magnetic Resonance; Canabinol. 


\section{Introdução}

A Cannabis é uma planta pertencente à família Cannabaceae e compreende três espécies principais: Cannabis sativa L., Cannabis indica e Cannabis ruderalis, sendo esta última incomum. ${ }^{1,2}$. Tratando-se da função narcótica, a partir da planta são preparados alguns derivados (preparações), como marijuana, haxixe e óleo de haxixe, que contêm compostos químicos provenientes da erva, denominados canabinoides, aos quais são atribuídas as propriedades psicotrópicas que classificam esses derivados como entorpecentes. ${ }^{3}$

Droga, segundo a Organização Mundial de Saúde (OMS), "é toda a substância que introduzida no organismo vivo modifica uma ou mais das suas funções".5 De acordo com a principal ação produzida no Sistema Nervoso Central (SNC), as drogas são classificadas em três grupos: depressoras, estimulantes e perturbadoras (ou modificadoras) do SNC. ${ }^{4}$

A maconha é classificada como perturbadora do sistema nervoso central, e pode-se dizer que é a droga bruta derivada da planta Cannabis sativa L. por tratar-se das flores e folhas secas desta planta. Ela é encontrada no mercado ilegal inalterada, bruta, prensada em tabletes e é comumente fumada.

Nos últimos anos tem-se discutido bastante sobre os efeitos medicinais da Cannabis, devido ao seu potencial terapêutico promissor, por exemplo, no tratamento de cólicas menstruais e convulsões, amígdalas inflamadas, enxaquecas e dores de cabeça, glaucoma e asma, no alívio da dor, etc. Dentre seus componentes estão quase todos os tipos de compostos químicos existentes, como por exemplo, mono e sesquiterpenos, açúcares, esteroides, flavonoides, hidrocarbonetos, compostos nitrogenados, etc. ${ }^{3}$

São 70 os canabinoides conhecidos e estes podem ser divididos em diferentes classes (tipos): Canabigerol (CBG), Canabicromeno (CBC), Canabidiol (CBD), $\Delta^{9}-$ tetrahidrocanabinol $\left(\Delta^{9}-\mathrm{THC}\right)$, Canabiciclol (CBL), Canabielsoin (CBE), Canabinol (CBN), Canabinodiol (CBND), Canabitriol (CBT) e outros variados. Dentre todos os existentes, os constituintes ativos, como por exemplo,
$\Delta^{9}$-THC, Canabinol, Canabidiol, etc., são considerados os principais canabinoides, ou por apresentarem importância forense ou de uso clínico. O isolamento de substâncias químicas presentes em drogas ilícitas, como é o caso dos canabinoides na maconha, é uma área importante no âmbito forense.

Focando-se nos compostos mencionados, seu isolamento se constitui em uma difícil tarefa devido à sua complexidade e também à presença de isômeros entre os constituintes dessa classe. Neste trabalho, algumas das técnicas analíticas citadas foram utilizadas na identificação e no isolamento de cannabinoides, como por exemplo, a RMN e a CG-EM. A Ressonância Magnética Nuclear é uma técnica que possui diversas aplicações: é utilizada no estudo da mobilidade e flexibilidade de moléculas, em cinética de reações químicas, na caracterização estrutural de sólidos, na obtenção de imagens internas de amostras, na identificação e elucidação estrutural de substâncias, etc., sendo esta última aplicação a que será utilizada no presente trabalho, com a finalidade de identificar e elucidar estruturalmente os principais canabinoides presentes nas amostras de maconha a serem estudadas. A cromatografia é um método físico-químico de separação de compostos presentes em misturas. Envolve a distribuição dos mesmos entre duas fases, uma móvel e outra estacionária. As duas fases podem ser de diferentes naturezas, e é possível combiná-las de diversas maneiras, o que gera diferentes tipos de métodos cromatográficos, como por exemplo, as cromatografias líquido-sólido, líquido-líquido, gás-sólido e gás-líquido, as duas últimas englobando a Cromatografia Gasosa (CG). A combinação da cromatografia gasosa com a espectrometria de massas é conhecida como CG-EM e permite a identificação de quase todos os compostos presentes em uma amostra.

\section{Metodologia}

A metodologia que foram empregadas neste trabalho dividiram-se em: obtenção dos extratos das amostras de maconha, preparo das amostras e obtenção dos espectros de $\mathrm{RMN}$ de ${ }^{1} \mathrm{H}$ das mesmas para posterior aplicação de 
métodos cromatográficos para a separação dos constituintes do extrato. Para a obtenção das amostras, a UFES possui um convênio com a Polícia Civil do Estado do Espírito Santo.

As análises de Ressonância Magnética Nuclear foram realizadas no NCQP (DQUI/UFES), em um espectrômetro Varian $400 \mathrm{MHz}$ (modelo VNMRS 400), operando com um campo magnético de ${ }^{9,4}$ Tesla e utilizando-se uma sonda de deteç̧ão direta de $5 \mathrm{~mm}$ BroadBand 1H/19F/X em diferentes solventes.

\section{Resultados e Discussão}

Após a extração por maceração de apreensões de maconha (150 mg) em $1 \mathrm{~mL}$ de metanol, o solvente foi evaporado e o resíduo submetido à análises de RMN. Após realizadas as análises de RMN de ${ }^{1} \mathrm{H}$, o extrato foi misturado em um balão e o solvente evaporado em evaporador rotativo, gerando-se um novo extrato bruto. Com esse extrato bruto da maconha foi feito uma Cromatografia em coluna tendo sílica-gel como fase estacionária e uma mistura de éter de petróleo e éter etílico (8:2), como fase móvel. Foram coletadas 52 frações, as quais após análise de placas de Cromatografia em Camada Delgada (CCD), foram agrupadas em cinco frações finais. O solvente destas cinco frações foram evaporadas e o resíduo obtido foi analisado em um cromatógrafo gasoso (CG-EM). A CG-EM, por permitir a rápida identificação de compostos presentes em misturas, foi então utilizada para complementar as informações obtidas por RMN. Para preparar suas soluções a serem injetadas no $\mathrm{CG}$, foi pesado uma quantidade do precipitado de cada fração e adicionado diclorometano grau HPLC em cada uma delas, de forma que a concentração em cada frasco ficasse entre 900 ppm e 1400 ppm. Foram realizadas 5 injeções, uma para cada fração, e analisados os cromatogramas de cada uma separadamente. Feito isto, constatou-se que apenas duas dessas frações apresentavam picos que foram identificados como canabinoides pela biblioteca NIST. Como no presente trabalho o interesse é nestes constituintes, somente os picos relativos a eles serão demonstrados. Esses picos foram encontrados nas frações 2 e 3 e seus cromatogramas estão representados nas figuras abaixo.

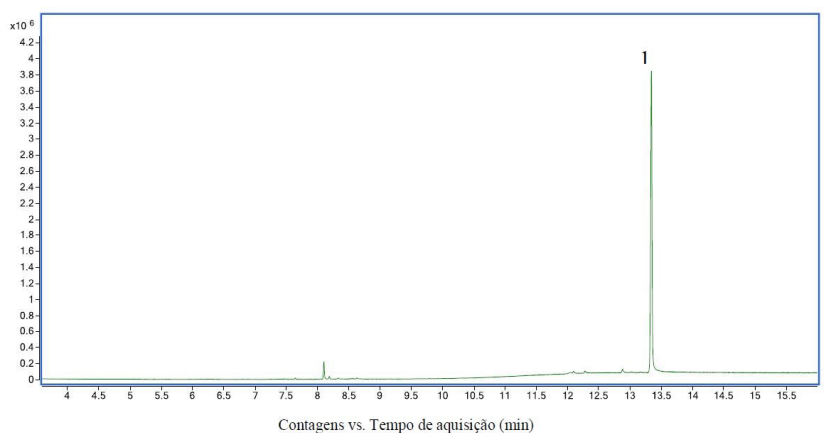

Figura 1. Cromatograma gasoso da fração 2.

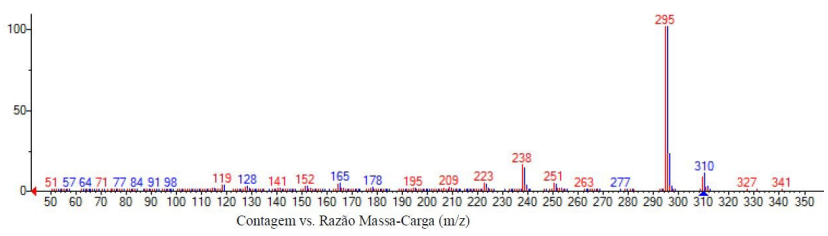

Figura 2. Perfil de fragmentação do canabinoide presenta na fração 2.

Por CG-EM, constata-se a presença de 2 compostos na fração 2, sendo um deles pertencentes à classe dos canabinoides. O pico 1 representado na Figura 1 refere-se ao CBN com $94,6 \%$ de probabilidade de ser este composto pela biblioteca NIST.

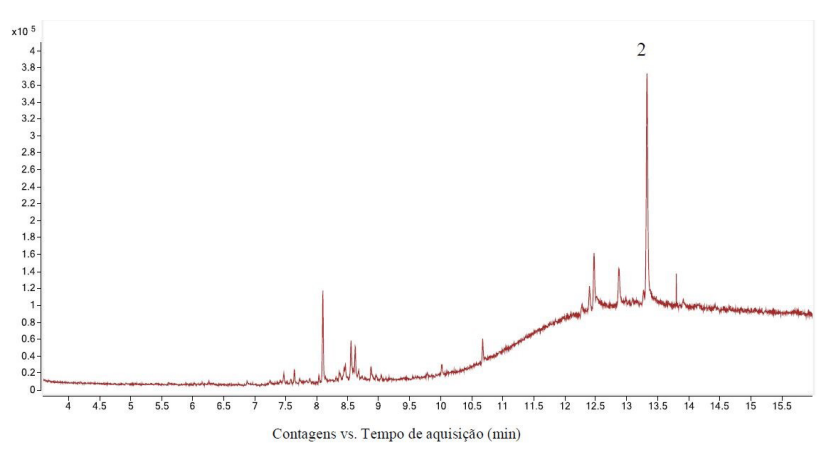

Figura 3. Cromatograma gasoso da fração 3. 


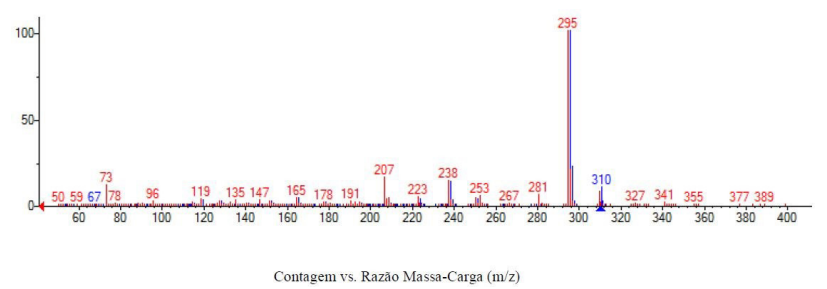

Figura 4. Perfil de fragmentação do canabinoide presenta na fração 3.

Cada pico mostrado no cromatograma da Figura 3 é referente a um composto, porém de acordo com a biblioteca NIST, apenas um deles pertence à classe dos canabinoides. O pico marcado com o número 2 na Figura 3 refere-se ao CBN com $81,9 \%$ de probabilidade.

Os demais picos, em todas as 5 frações, não foram identificados como canabinoides pela biblioteca NIST, ou por realmente não serem canabinoides ou por eles não estarem registrados nela. O composto designado como sendo o CBN foi então submetido às análises de RMN e o espectro de RMN de ${ }^{1} \mathrm{H}$ está representado na Figura 5, abaixo. Os sinais referentes aos hidrogênios do composto isolado são coincidentes com os sinais apresentados na literatura e com estudos anteriores realizados pelo nosso grupo de pesquisa.

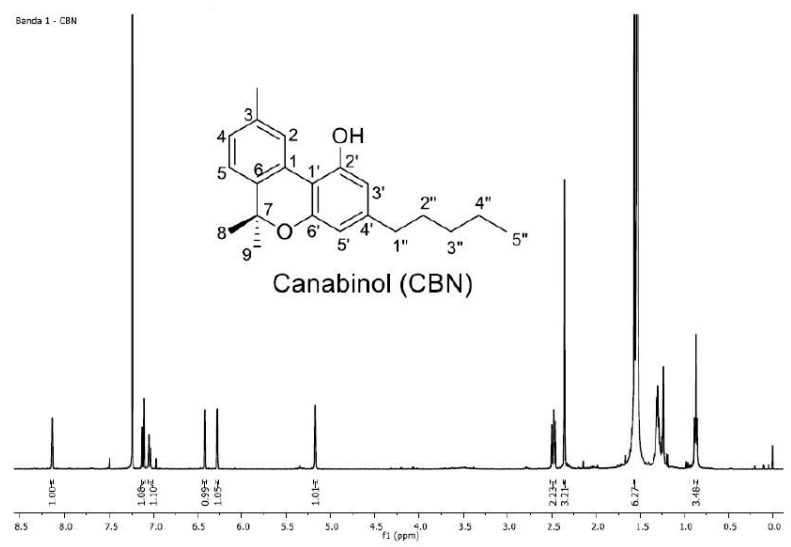

Figura 5. Espectro de $\mathrm{RMN}$ de ${ }^{1} \mathrm{H}$ do canabinol (CBN).

\section{Conclusões}

Neste estudo, amostras de maconha apreendidas pela PC-ES foram analisadas por cromatografia e por espectroscopia de RMN de ${ }^{1} \mathrm{H}$. Primeiramente, testes de extração foram realizados com uma amostra para se chegar a um método satisfatório e com boa relação custo benefício. $\mathrm{O}$ método escolhido utilizou metanol como solvente extrator, maceração como método de extração e clorofórmio deuterado para as análises de RMN de $1 \mathrm{H}$ dos extratos obtidos.

Os extratos brutos não foram analisados separadamente neste estudo, uma vez que se pretende primeiramente isolar e identificar os constituintes obtidos das apreensões de maconha. Todas as extrações foram agrupadas e o novo extrato gerado foi submetido a cromatografia, dando origem a 5 frações cromatográficas. Após análise por CG-EM, constatou-se que o CBN estava presente em duas dessas frações.

Para as duas técnicas, separadamente, observou-se que as amostras não apresentam grandes diferenças e que o composto THC não foi observado, mostrando o grau de degradação das amostras apreendidas.

\section{Agradecimentos}

Agraço a Deus, à UFES, ao LabPetro e a Polícia Civil, que permitiu a realização dos trabalhos.

\section{Referências Bibliográficas}

1. La Paz, S. M.; Marín-Aguilar, F.; García-Giménez, M. D.; Fernández-Arche, M. A. Hemp (Cannabis sativa L.) Seed Oil: Analytical and Phytochemical Characterization of the Unsaponifiable Fraction. J. Agric. Food Chem., 62, 1105-1110, 2014.

2. Warf, B. High Points: An Historical Geography Of Cannabis. Geographical Review, 104 (4), 414-438, 2014.

3. Elsohly, M. A.; Slade, D. Chemical constituents of marijuana: The complex mixture of natural cannabinoids. Life Sciences, 78, 539-548, 2005.

4. Passagli, M. F. Toxicologia Forense: Teoria e Prática. $4^{\mathrm{a}}$ Ed. Campinas: Millennium, 2013. 515 p.

5. Associação Humanidades. Manual de Prevenção do Uso de Drogas 


\title{
Gabriel S. Ferreira', Júlia A. Leite', Bianca B. Merlo², Valdemar L. Junior', Wanderson Romão ${ }^{3}$ \& Alvaro C. Neto ${ }^{1 *}$
}

\author{
${ }^{1}$ Departamento de Química, Universidade Federal do Espírito Santo, Av. \\ Fernando Ferrari, 514. Vitória - ES, Brasil. \\ ${ }^{2}$ Laboratório de Química Legal, Superintendência de Polícia Técnico- \\ Científica da Polícia Civil do Estado do Espírito Santo, Av. Nossa Senhora \\ da Penha, 2290. Vitória - ES, Brasil \\ ${ }^{3}$ Instituto Federal do Espírito Santo, Av. Ministro Salgado Filho, 1000. \\ Vila Velha - ES, Brasil. \\ *E-mail: alvarocunhaneto@gmail.com
}

